\section{Group A streptococcal carriage among residents of an urban homeless shelter}

\section{To the Editor:}

Population reservoirs of asymptomatic group A streptococcal (GAS) carriage have been linked to outbreaks of active GAS infection in close contacts (1). The prevalence of GAS carriage is $1 \%$ to $2 \%$ among adults in the general population and greater than $5 \%$ among those in close contact with individuals with active GAS infection (2-5).

Residents of homeless shelters may represent a population at increased risk of GAS carriage and infection. Homeless persons commonly have health conditions, such as lung disease and alcohol abuse, that are potential risk factors for severe GAS infection $(4,6)$, and the possible spread of infections among homeless persons congregating in shelters is a serious concern. A recent study (7) conducted in Barcelona, Spain, found that invasive GAS infections were more common among injection drug users who were homeless than those who were housed. However, no published studies have examined GAS carriage in a representative sample of homeless people. Therefore, we determined the prevalence of GAS carriage in a representative sample of residents at a 600 -bed shelter for single adult men in Toronto, Ontario.

Between 1992 and 1995, the census tract containing the shelter reported the highest incidence of invasive GAS infections in Ontario (unpublished data). In addition, seven of 15 infections due to $\mathrm{M}$ nontypeable $\mathrm{T}$ type 5/27/44 Streptococcus pyogenes in Ontario between 1992 and 1995 occurred in a 12-month period in men who were either homeless or had addresses in census tracts proximate to the shelter; five of seven men were known to abuse alcohol and/or use intravenous drugs, suggesting circulation of a clone. In March 2000, nose, throat and skin swab specimens were obtained from a convenience sample of consecutive consenting shelter residents who presented at the shelter medical clinic with skin lesions. Of the nine residents tested, three had GAS isolated from skin lesions (one also had GAS isolated from a nasal swab), and one had GAS isolated from both throat and nose swabs. Pulsed field gel electrophoresis revealed two pairs of residents who were colonized with strains of identical pattern.

In July and August 2003, shelter residents were randomly selected to take part in a microbiological survey. Data collection consisted of a face-to-face interview and collection of nasal, throat and skin lesion swabs. Interview questions determined the prevalence of potential risk factors for GAS carriage, including duration of homelessness, prevalence of chronic medical conditions and recent use of injection drugs.
Information on current prescription medication use and antibiotic use within the past three months was obtained. The Cutting down, Annoyance by criticism, Guilty feeling, and Eye-openers (CAGE) questionnaire was used to screen for alcoholism (8); two or more affirmative answers were considered to be indicative of probable alcohol abuse or dependence. Swab cultures from the nose and throat were collected from each participant using the Copan Venturi Transystem (Copan Diagnostics Inc, USA). If skin lesions were present, the largest area of open skin was swabbed. Swabs were planted within $8 \mathrm{~h}$ of collection and processed using standard methodology with latex agglutination for streptococcal typing (Prolab Technologies Inc, Canada) (12). Confirmed GAS isolates were sent to the Canadian National Centre for Streptococcus (Edmonton, Alberta) for $\mathrm{M}$ serotyping and $\mathrm{T}$ agglutination typing. The study was approved by the St Michael's Hospital (Toronto, Ontario) research ethics board.

Based on a one-sample binomial test, it was determined that 233 homeless subjects would be required to detect, with $80 \%$ power, an estimated $5 \%$ carriage prevalence in homeless people relative to a $2 \%$ level in the general adult population. Confidence interval estimation for binomial proportions was used to determine whether the carriage prevalence among the homeless differed significantly from the general population. Exact estimation methods were applied to logistic regression analysis using SAS version 8.2 (SAS Institute Inc, USA) to estimate the association between potential risk factors for GAS carriage and carriage status.

Of 268 shelter residents who were contacted and eligible, 243 agreed (91\% response rate) to participate in the study. The mean \pm SD age of participants was $43 \pm 10$ years, and the mean duration of the current episode of homelessness was $36 \pm 60$ months. Chronic health conditions were reported at the following rates: asthma (17\%), chronic obstructive pulmonary disease $(13 \%)$, hepatitis C $(9 \%)$, diabetes $(8 \%)$, eczema (6\%), psoriasis (5\%) and cancer (2\%). Injection drug use within the past 30 days was reported by $4 \%$, and alcoholism (based on the CAGE score) was identified in $52 \%$ of participants.

Five individuals (2.1\% of participants; $95 \%$ CI 0.9 to 4.7) were identified as GAS carriers through positive throat swabs. No nasal or skin lesion swabs were positive for GAS. The five carriers were found to have five different GAS serotypes (M2T2, M12T12, M73T3, M75T25 and M81T3). Antibiotic use within the past 90 days was reported by 24\% of participants, none of whom were GAS carriers. In univariate analyses, only age was significantly associated with GAS carriage: participants in the lowest age quartile (younger than 37 years) were 11.5 times more likely to be carriers than those 37 years of age or older $(\mathrm{P}<0.04)$. The presence of an underlying chronic condition, alcohol use, 
injection drug use and duration of time spent homeless were not associated with GAS carriage.

In conclusion, the observed $2.1 \%$ prevalence of GAS carriage among a representative sample of men residing in a homeless shelter is comparable with that typically reported in the general adult population $(2,3)$. In addition, there was no clustering of GAS serotypes to suggest GAS transmission between residents. Certain factors may help to explain these results. The March 2000 pilot study found high rates of GAS carriage among individuals with symptomatic skin lesions, whereas the follow-up microbiological survey enrolled primarily asymptomatic individuals. In addition, although no season-dependent variation in the level of GAS carriage has been demonstrated $(2,3)$, it is possible that such variation exists and that carriage rates among shelter residents may be higher during the winter months, when residents congregate indoors for longer periods of time.

The anatomical site of GAS carriage may be a significant factor in transmission. All five carriers identified in the follow-up study had GAS isolated exclusively from throat specimens. However, it has been suggested that nasal carriers are more likely to spread infection (9). In addition, outbreaks of severe infection in nursing homes and hospitals have implicated skin lesions as common foci of infection (10). Indeed, GAS isolates obtained from skin lesions were involved in each matching serotype pair in the original pilot study. Thus, future research should focus on GAS carriage among shelter residents during the winter months or among those with symptomatic skin lesions.

ACKNOWLEDGEMENTS: The authors thank the staff at Seaton House (Toronto, Ontario) for their time and support, and Vanessa Porter for her key role in helping to analyze swab specimens.
Gordon JM Bargh MSc Faculty of Medicine, University of Toronto Toronto, Ontario

Jeffrey S Hoch PhD, Stephen W Hwang MD Centre for Research on Inner City Health, St Michael's Hospital, Toronto, Ontario

Mark Speechley $\mathrm{PhD}$ Department of Epidemiology and Biostatistics, University of Western Ontario, London, Ontario

Barbara M Willey MLT ART, Allison McGeer MD, Shared Department of Microbiology, Toronto Medical Laboratories/Mount Sinai Hospital, Toronto, Ontario

\section{REFERENCES}

1. Sherertz RJ, Bassetti S, Bassetti-Wyss B. "Cloud" health-care workers. Emerg Infect Dis 2001;7:241-4.

2. Strömberg A, Schwan A, Cars O. Throat carrier rates of beta-hemolytic streptococci among healthy adults and children. Scand J Infect Dis 1988;20:411-7.

3. Hope-Simpson RE. Streptococcus pyogenes in the throat: A study in a small population, 1962-1975. J Hyg (Lond) 1981;87:109-29.

4. Davies HD, McGeer A, Schwartz B, et al. Invasive group $A$ streptococcal infections in Ontario, Canada. Ontario Group A Streptococcal Study Group. N Engl J Med 1996;335:547-54.

5. Falck G, Holm SE, Kjellander J, Norgren M, Schwan A. The role of household contacts in the transmission of group A streptococci. Scand J Infect Dis 1997;29:239-44.

6. Hwang SW, Dunn JR. Homeless people. In: Galea S, Vlahov D, eds. Handbook of Urban Health: Populations, Methods, and Practice. New York: Kluwer/Plenum, 2005:19-41.

7. Sierra JM, Sanchez F, Castro P, et al. Group A streptococcal infections in injection drug users in Barcelona, Spain. Medicine 2006;85:139-46.

8. Ewing JA. Detecting alcoholism. The CAGE questionnaire. JAMA 1984:252:1905-7.

9. Hamburger M Jr, Green MJ, Hamburger VG. The problem of the "dangerous carrier" of hemolytic streptococci. J Infect Dis 1945;77:68-81.

10. Schwartz B, Elliott JA, Butler JC, et al. Clusters of invasive group A streptococcal infections in family, hospital, and nursing home settings. Clin Infect Dis 1992;15:277-84.

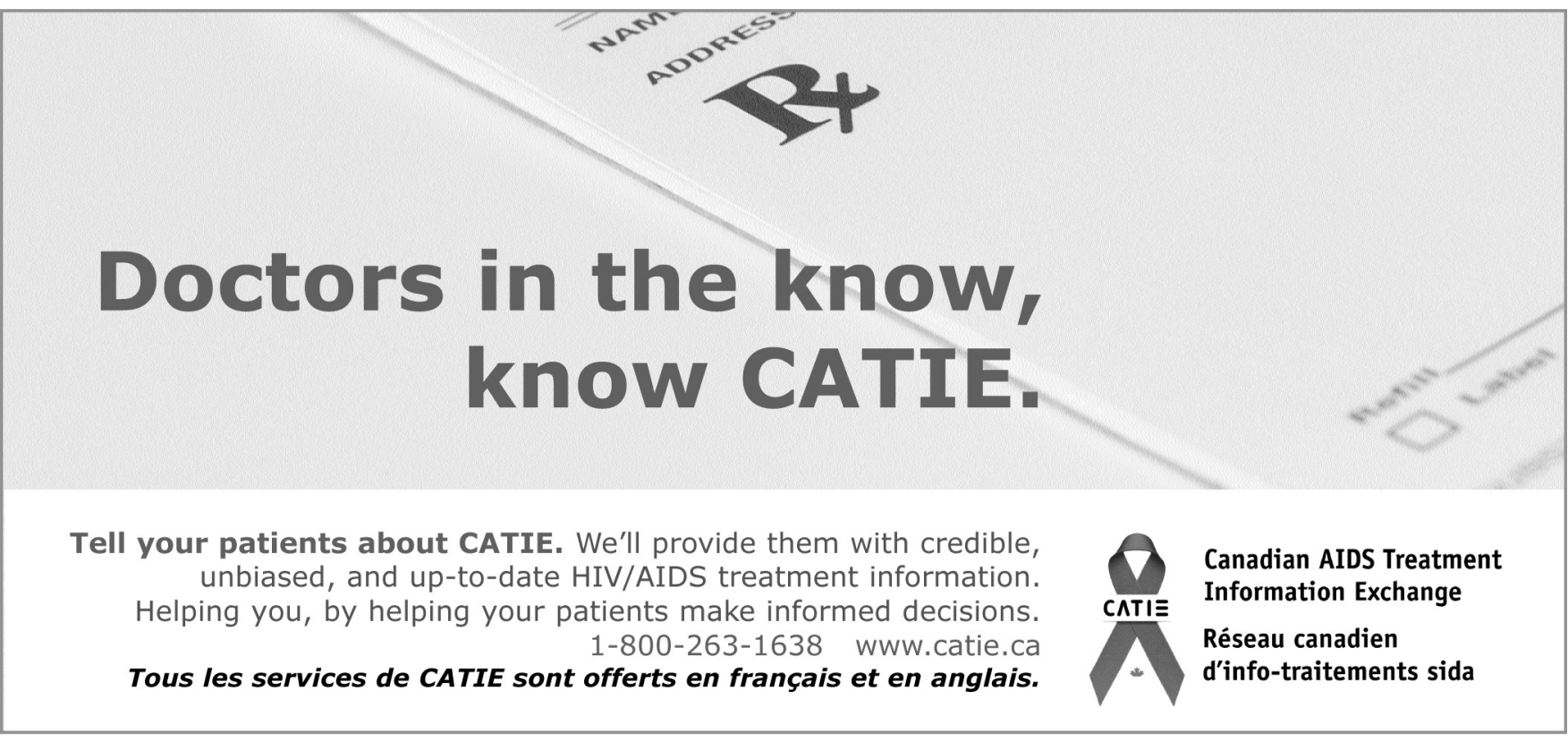




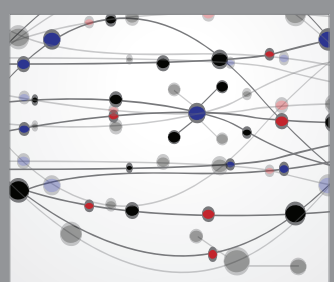

The Scientific World Journal
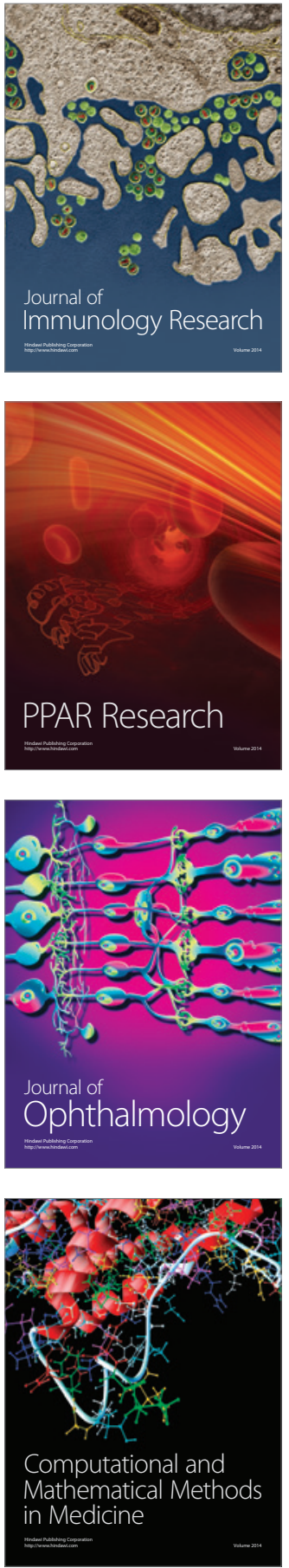

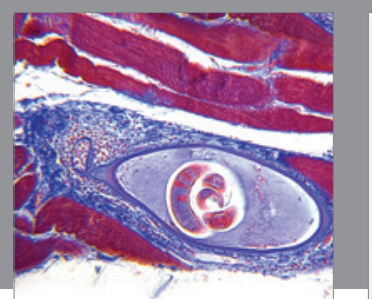

Gastroenterology Research and Practice

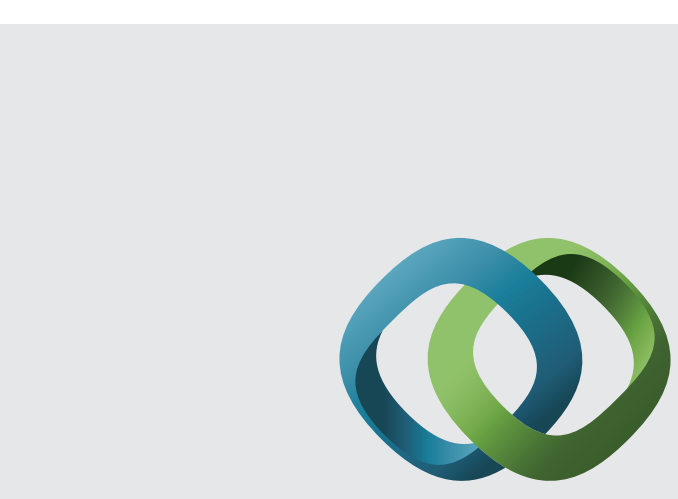

\section{Hindawi}

Submit your manuscripts at

http://www.hindawi.com
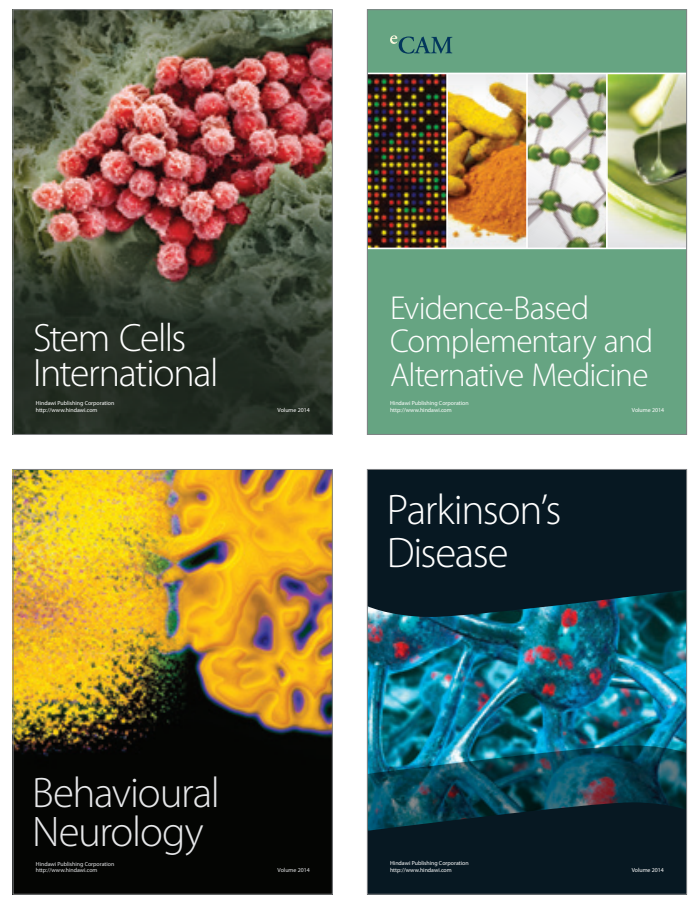
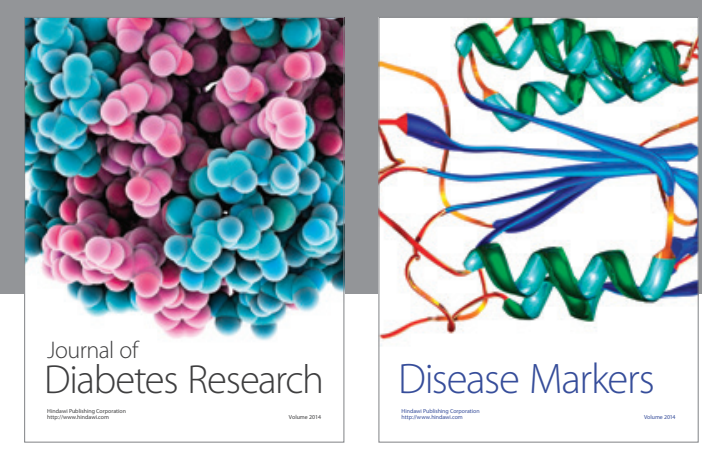

Disease Markers
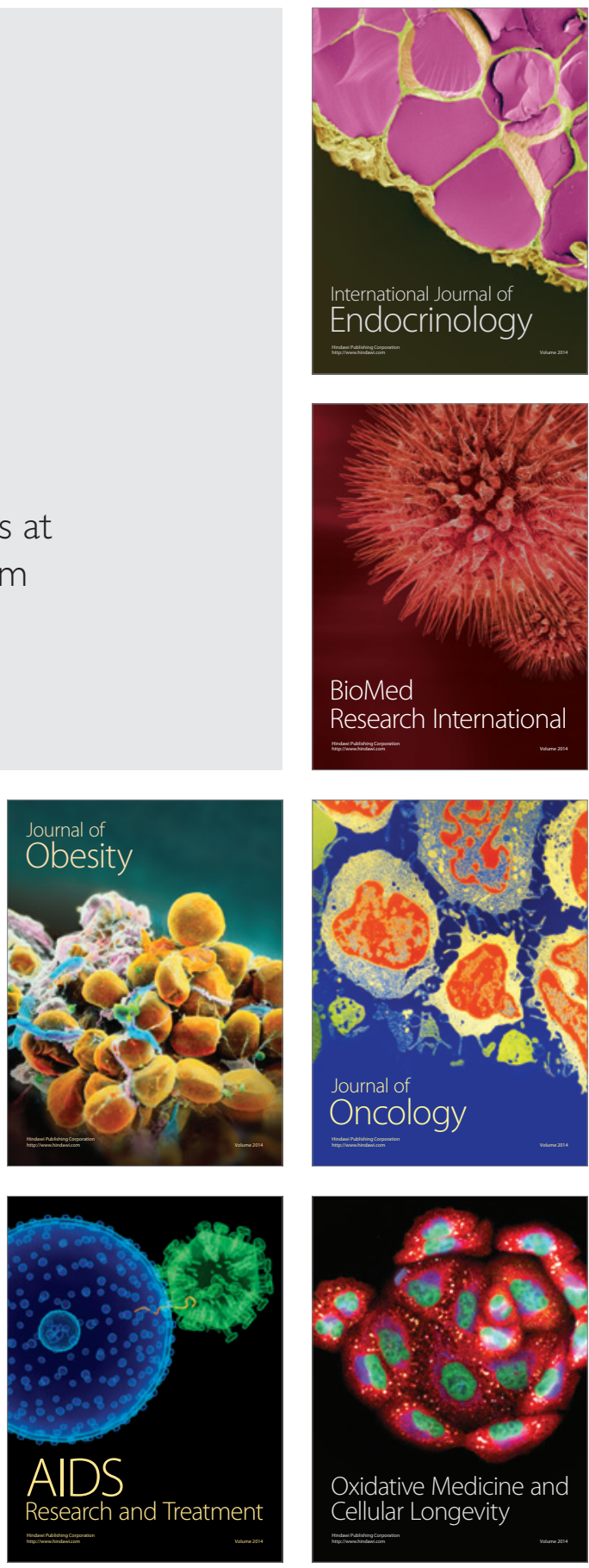